11 | 2007

Varia

\title{
Les conversions de cens au Moyen Âge
}

Auxerre, 27-28 octobre 2006

\section{Laurent Feller}

\section{(2) OpenEdition}

Journals

Édition électronique

URL : https://journals.openedition.org/cem/1123

DOI : $10.4000 /$ cem. 1123

ISSN : 1954-3093

Éditeur

Centre d'études médiévales Saint-Germain d'Auxerre

Édition imprimée

Date de publication : 15 août 2007

ISSN : 1623-5770

Référence électronique

Laurent Feller, "Les conversions de cens au Moyen Âge », Bulletin du centre d'études médiévales

d'Auxerre | BUCEMA [En ligne], 11 | 2007, mis en ligne le 30 août 2007, consulté le 22 septembre 2022.

URL : http://journals.openedition.org/cem/1123; DOI : https://doi.org/10.4000/cem.1123

Ce document a été généré automatiquement le 22 septembre 2022.

\section{c) (i) (2) (2)}

Creative Commons - Attribution - Pas d'Utilisation Commerciale - Partage dans les Mêmes Conditions 4.0 International - CC BY-NC-SA 4.0

https://creativecommons.org/licenses/by-nc-sa/4.0/ 


\section{Les conversions de cens au Moyen Âge}

Auxerre, 27-28 octobre 2006

\section{Laurent Feller}

1 Dans le cadre du programme «La circulation des richesses au Moyen Âge », le Lamop (UMR 8589, CNRS/Paris 1) a organisé à Auxerre, les 27 et 28 octobre 2006, un séminaire international portant sur la question des conversions de cens. La réunion était abritée dans les locaux du Centre d'études médiévales que nous avons plaisir à remercier de son hospitalité généreuse.

2 Laurent FELLER présentant l'introduction résume la problématique du sujet. Cette question a été abordée brièvement par Georges Duby qui, dans L'économie rurale et la vie des campagnes a imposé l'idée que, à partir du XII ${ }^{\mathrm{e}}$ siècle et jusqu'au XIV ${ }^{\mathrm{e}}$ siècle, les redevances en argent s'étaient imposées au détriment des redevances en nature et en travail. Ce processus aurait compté pour beaucoup dans l'érosion des revenus seigneuriaux. Or, l'observation ne peut être généralisée. En 1957, par exemple, Rosario Romeo remarquait, à Origgio, près de Milan, un processus inverse, puisque les redevances en nature s'imposaient à la fin du XII siècle. De même, les travaux de $\mathrm{J}$. Ambrose Raftis et de son école montraient que l'inflation anglaise de la fin du XII siècle avait entraîné un retour soit au prélèvement en nature soit une transformation profonde du système de production. Enfin, dans les années 1980-1990, les travaux portant sur la seigneurie en Italie montraient, au XIII siècle, selon une chronologie et des modalités pouvant encore être précisées, une tendance des seigneurs à asseoir leurs revenus sur des redevances fixes en nature établies à un niveau élevé.

La réflexion sur ce point peut et doit progresser. Elle implique de s'interroger sur la capacité des seigneurs à agir comme des acteurs économiques rationnels, susceptibles de définir un but et de se donner les moyens de l'atteindre et à effectuer des choix qui font sens. Elle implique aussi de s'interroger sur le rapport entretenu par les seigneurs avec les marchés des denrées agricoles. 
4 La question a semblé d'un intérêt suffisant pour rassembler une dizaine de chercheurs venus de France, d'Italie, d'Angleterre, d'Espagne et d'Allemagne pour deux journées de travail qui se sont avérées intenses et, on l'espère, fructueuses.

5 En Allemagne, au $\mathrm{XV}^{\mathrm{e}}$ siècle, dans la région de Nüremberg, des cueilloirs permettent d'étudier dans le détail les versements effectués par les paysans (Julien DEMADE). La question des conversions est d'importance, entre autres parce que les redevances sont mal perçues et que les retards de paiement y sont structurels. Elles sont fréquemment converties, de l'argent étant le plus souvent accepté en lieu et place de la redevance en nature normalement exigible. Il arrive aussi que du travail soit proposé par les paysans en cas de non versement des redevances. Les versements se font donc ainsi par conversion de la valeur du produit demandé soit en argent soit en travail, ce qui pose la question du rôle du marché dans cette opération. Les différentes céréales produites par les tenures et exigées par les seigneurs n'ont pas nécessairement le même intérêt commercial.

6 Pour l'Angleterre, la période qui va de 1170 à 1220 s'avère cruciale (Philipp schofIELD). C'est celle durant laquelle s'établit le high farming, c'est-à-dire celle de l'exploitation seigneuriale directe des domaines. Les sources toutefois montrent une difficulté majeure en ceci qu'elles informent bien sur les tenures libres durant le XII ${ }^{e}$ siècle et sont peu disertes sur les tenures serviles et que, en revanche, au XIII ${ }^{e}$ siècle, elles disent beaucoup sur les tenures serviles et peu sur les tenures libres. Il est fréquent que les redevances en nature soient converties en redevances en argent. Les corvées, pour leur part, peuvent être en partie substituées par un versement en argent. Enfin, les rentes en argent se multiplient à partir du XIII ${ }^{e}$ siècle, au fur et à mesure du retrait de seigneurs de l'exploitation directe.

7 Une comparaison entre l'Angleterre et la Normandie (Catherine LETOUZEY) à travers la documentation de la Sainte-Trinitié de Caen montre en revanche une grande stabilité dans la structure des redevances, les prestations en nature et en travail demeurant alors, dans les deux zones géographiques, majoritaires, sans altération. Ce non-choix lui réussit bien, puisque, aux XIII ${ }^{e}$ et XIV ${ }^{e}$ siècles, les liquidités ne lui font jamais défaut.

Prenant les choses d'un tout autre point de vue, Isabelle THEILLER montre, en commentant un chirographe de 1209, que la valeur des biens sur lesquels sont assises les rentes en nature est connue grâce à une pratique normale des relations de marché. Les montants ne sont donc pas arbitraires et peuvent donner lieu à des calculs de la part des acteurs.

Dans les pays de l'Ouest de la France étudiés par Daniel ріснот, le choix est précoce et massif. À partir du XI ${ }^{\mathrm{e}}$ siècle, les seigneurs exigent des cens fixes et lourds, mais en argent, les versements en nature étant très rares. Ce sont les conditions techniques de la mise en valeur qui expliquent ce fait: le seigneur évite que lui soient remises des céréales de peu de valeur ; surtout, dans une région où la jachère est très longue, le prélèvement en argent assure un revenu même quand la terre ne porte pas de fruits. Seules les terres récemment défrichées et d'un haut rapport versent des cens en nature. Les comptabilités des châtelains des comtes de Savoie aux XIII ${ }^{e}-\mathrm{XV}^{e}$ siècles (Nicolas CARRIER) montrent une préférence marquée des princes pour les redevances fixes en nature, principalement spécifiées en céréales. Elles ne sont cependant pas versées directement, les châtelains étant dans l'obligation de les racheter à titre personnel et donc de verser de l'argent au lieu des céréales. Cette manipulation fait des châtelains 
des agents particulièrement actifs sur le marché des céréales, cela durant une période où l'offre est déficitaire. Le processus de formation des prix de ces céréales est particulièrement intéressant à étudier, dans la mesure où il diffère sensiblement du prix de marché et où leur niveau est lié à des considérations sociales et politiques davantage qu'économiques.

11 Étudiant un acte paradoxal, la conversion d'une rente en argent en une rente en vin en faveur du couvent des frères Mineurs d'Avignon en 1368, Catherine LENOBLE s'interroge sur les motivations de ce versement qui contraint les Mendiants à devenir des acteurs sur le marché, la quantité concernée étant trop importante pour correspondre à la seule consommation du couvent. La rente ainsi offerte est un droit sur un bien, non un usage ou une propriété. Elle est également légitime parce qu'elle dispense le donateur d'une transaction. Elle apparait aussi comme un choix spirituel de la part du donateur dont les implications socio-économiques sont complexes et nombreuses.

12 Luigi PROVERo, pour sa part, choisit de considérer les chartes de franchise piémontaise sous l'angle de la conversion. Les franchises en effet transforment les anciennes coutumes, en permettant au concessionnaire de recevoir une somme fixe annuelle dont le versement est le signe de la redéfinition des rapports entre la communauté et le seigneur. C'est de la structure même du prélèvement qu'il s'agit alors : le seigneur peut à ce moment précis l'adapter à ses besoins et, s'il exige aussi à ce moment le paiement d'un fort droit, il peut en profiter, le cas échéant pour se désendetter. La conversion apparaît comme un moment privilégié dans la relation seigneurs-paysans dans la mesure où elle contraint à négocier.

13 Le Latium propose une autre situation où la conversion est, aux XIII ${ }^{e}$ et XIV ${ }^{e}$ siècles, globale (Sandro CAROccI). Il ne s'agit pas là, cependant, de négocier les relations entre la communauté juridique et son seigneur, mais de redéfinir les fondements mêmes de l'exploitation à travers l'imposition du ius serendi, du droit de semer. Le ius serendi apparaît dans le Latium au XIII ${ }^{\mathrm{e}}$ siècle. Il repose sur une réappropriation du sol par le seigneur qui devient le seul propriétaire de terre céréalicole. Il doit donner celle-ci à cultiver aux paysans du village concerné et ceux-ci sont dans l'obligation de les mettre en valeur. Tous les ans le seigneur procède à une réattribution des champs, chacun recevant un lot en fonction de l'importance de sa famille et du nombre de bœufs et de charrue possédés : il n'y a donc pas de tenures. L'exemple des statuts de Genazzano de 1379 montre que l'apparition du ius serendi correspond à une réorganisation profonde des exploitations agraires et qu'elle permet à l'élite paysanne de consolider sa position dans l'habitat.

14 Autre situation de conversion globale, celle de la Navarre (Carlos LALIENA). Les redevances payées par les serfs, les pechas sont, aux $\mathrm{XI}^{\mathrm{e}}$-XII ${ }^{\mathrm{e}}$ siècles, prélevées en nature. Souvent légères, elles font l'objet, à partir de 1180, de conversions de la part des souverains qui les remplacent par une redevance unique exigée désormais en argent. La conversion s'effectue de deux façons différentes. Soit chaque famille, quelle que soit sa richesse verse la même chose. Soit la communauté est taxée d'un certain montant et répartit celui-ci entre ses membres. Les souverains, de la sorte, favorisent la monétarisation de l'économie navarraise et contraignent, indirectement, les paysans à échanger sur les marchés. Dans les cas où ils continuent de prélever la pecha en céréale, ils tirent profit de l'activité accrue des marchés urbains. La conversion, ici, sert véritablement à moderniser la vie économique en promouvant les marchés et en améliorant la circulation monétaire. 
En Catalogne (Pere BENITo), les redevances «d'ancien style » des $\mathrm{X}^{\mathrm{e}}$ et $\mathrm{XI}^{\mathrm{e}}$ siècles peuvent être considérées comme lourdes. Il s'agit des agriers, tasques et champarts. Ils sont remplacés, au XII ${ }^{e}$ siècle, durant un moment de conjonctures particulièrement défavorables aux seigneurs par des redevances en nature, fixes et annuelles. Celles-ci entraînent une baisse des revenus seigneuriaux. Cette forme de paiement simplifie le prélèvement et diminue les frais de transport. Le système connaît de nouvelles oscillations au début du XIV ${ }^{\mathrm{e}}$ siècle.

Roland VIADER, enfin, présente les variations du système de prélèvement en Gascogne et dans le Toulousain où le système du casal s'appuie sur un type de prélèvement particulier, résumé dans la queste. L'ensemble est mis à mal par la construction de nouveaux habitats qui modifient en profondeur les règles du jeu du fait de l'apparition de nouveaux types de tenure.

7 Les actes du séminaire devraient être rassemblés au printemps 2007 pour une publication rapide.

Rappel du programme :

- Pere BeNITO (Lamop/Univ. Paris 1) : «Ad censum reducere ». Polysémie des transformations du prélèvement coutumier dans la seigneurie foncière catalane (XIIe-XIVe siècles).

- Sandro CARocci (Université de Rome 2) : Les conversions de cens en Italie méridionale (XIIeXIII siècle).

- Nicolas CARRIER (Université de Lyon 3) : Les rachats de redevances en nature en Savoie du Nord à la fin du Moyen Âge.

- Julien DEMADE (CNRS/LAMOP) : Les versements des tenanciers d'un village franconien du XVe siècle, ou la conversion systématique de la norme seigneuriale.

- Chris DYER (Université de Leicester) : Conversion of rents in kind and in labour into cash in England, 10th-13th centuries.

- Carlos LALIENA (Université de Saragosse) : Les conversions de cens en Aragon et en Navarre (1180-1230).

- Clément LENOBLE (Lyon 2/EHESS) : Comment l'argent se changeait-il en vin ? Un accord entre les Sade et les frères Mineurs d'Avignon à la fin du XIVe siècle.

- Catherine letouzey (Université de Paris 1) : Conversions de cens dans les domaines de la Sainte-Trinité de Caen en Angleterre et en Normandie.

- Daniel ріснот (Université de Rennes) : Cens de l'Ouest, cens en argent (XIe-XIIIe siècles).

- Luigi PRovero (Université de Turin) : Le trasformazioni del prelievo nel confronto tra signori e comunità (Piemonte meridionale, XII-XIII secolo).

- Phillipp sCHofield (Université de Aberyswyth) : Conversions de redevances en East Anglia.

- Roland VIADER (CNRS/Framespa) : Affranchissements et conversions du prélèvement dans le Sud-Ouest de la France.

\section{INDEX}

Mots-clés : cens 